\title{
A Case Report on Primary Neuroendocrine Cancer of Vagina
}

\author{
Kumari Puja*, Ashok Kumar Arya, Arun Yadav and Durgesh
}

S.N Medical College, Agra, India

*Corresponding author: Kumari Puja, Department of Radiation Oncology, S.N Medical College, Agra, India, Tel: 8240023414

\begin{abstract}
Neuroendocrine tumors of the gynaecologic tract are rare, and pose a significant clinical challenge because of the tumor heterogeneity and lack of standardized guidelines for treatment. Primary neuroendocrine tumor of vagina is extremely rare and only 26 cases have been reported so far in literature. Most, but not all, neuroendocrine tumors of the gynaecologic tract have an aggressive clinical course and clinically share similarities with small cell lung cancer. Cumulative data supports a multi-modality therapeutic strategy. A proposed management algorithm for neuroendocrine carcinomas of the vagina is outlined including surgery, radiotherapy and chemotherapy. We report a case of neuroendocrine cancer of vagina which we treated at our institution.
\end{abstract}

\section{Introduction}

Primary neuroendocrine small cell carcinoma of vagina is a very rare entity. Small cell carcinoma is characterized by its small cells with a scanty amount of cytoplasm and small hyperchromatic nuclei which are about 2-3 times the size of lymphocytes. Only $5 \%$ of small cell carcinomas arise in extrapulmonary sites [1]. They have histological, ultrastructural and immunohistochemical features similar to small cell carcinoma reported elsewhere in the body [2-4]. They have an aggressive clinical behaviour and poor prognosis [3,4]. Lack of clinical data and randomized controlled trials pose difficulty in establishment of standard treatment guidelines. Surgical resection is appropriate in selected cases. Radiotherapy along with etoposide/platinum-based chemotherapy is used for neuroendocrine carcinomas of vagina. We came across a case of primary neuroendocrine small cell carcinoma of vagina which we are reporting.

\section{Case}

A 50-years-old post-menopausal female, G2P2L2, presented to our OPD with the complains of intermittent bleeding per vagina for 2 months which was not associated with any foul smell but was associated with intermittent pain in lower abdomen. No associated comorbidities or no significant family history was found.

She underwent gynaecologic examination which showed a firm growth involving the upper third of vagina. Per rectal examination showed no involvement of the rectal mucosa or the parametrium. A punch biopsy was done which showed poorly differentiated malignant neoplasm. On microscopic examination highly cellular, irregular nests of tumor cells were seen surrounding the stroma. Also, sheets of monotonous round cells infiltrating the subepithelial tissue was seen. Overlying squamous epithelium was unremarkable. Overall morphology was suggestive of malignant round cell tumor. On IHC examination, tumor cells were immunopositive for $\mathrm{Ck}$ (dim positive), Synaptophysin (dim positive), chromogranin (dim positive). Ki67 index was $90 \%$. Tumor cells were immunonegative for LCA, CD20, Mic-2, Desmin, P63 and Vimentin.

Whole body pet scan showed FDG avid heterogeneously enhancing lesion in vagina, enlarged right external iliac lymph node and enlarged left internal iliac lymph node not separable from left adnexa. No lesions were seen in the lungs or any other site and there were no complains of flushing, diarrhoea, hypercalcemia, or hypoglycaemia. Hence, we made a diagnosis of non-functional Primary neuroendocrine carcinoma of vagina (Figure 1 ).

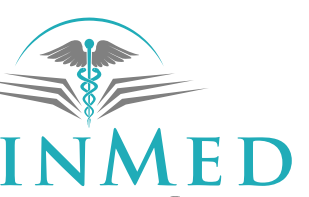

INTERNATIONAL LIBRARY 

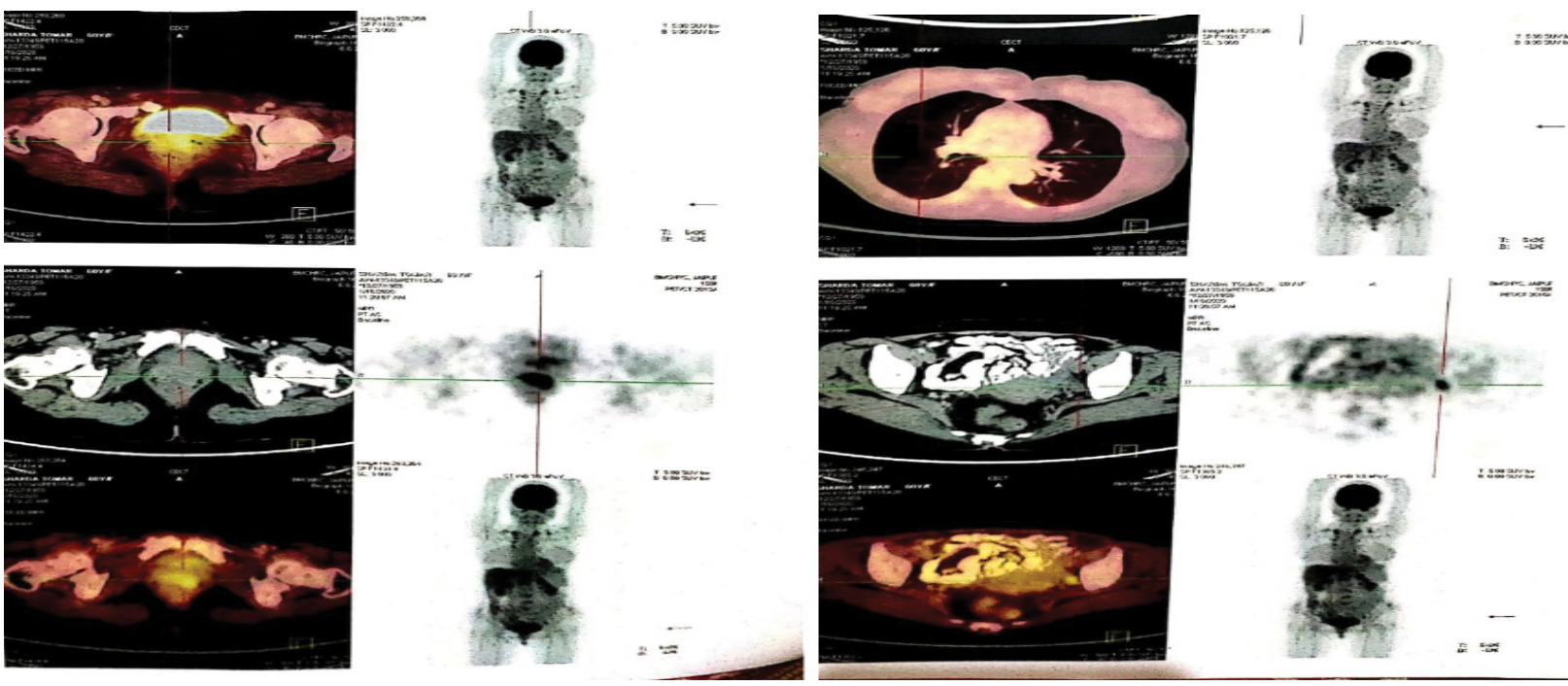

Figure 1: Pretreatment PETCT vs. PETCT at relapse.

The patient was sent for surgical opinion but could not be operated due to involvement of lymph nodes. The patient was planned for 6 cycles of combination chemotherapy with cisplatin and etoposide but she was not ready for long term treatment with chemotherapy. So, we treated her with EBRT to whole pelvis by APPA fields using cobalt 60 to a total dose of $50 \mathrm{~Gy}$ in 25 fractions, 5 fractions per week regimen.

She also received concurrent chemotherapy with weekly injection cisplatin $35 \mathrm{mg} / \mathrm{m}^{2}$ and injection etoposide $100 \mathrm{mg} / \mathrm{m}^{2}$ on D2, D4, D6 three weekly. She became asymptomatic after completion of the treatment. MRI of pelvis was done after 6 weeks of completion of treatment which showed no residual disease.

Patient was kept on monthly follow up after completion of the treatment. She was on regular follow up for three months but then defaulted for 8 months and then presented to us with complains of low back pain, generalised weakness, recurrent vomiting and pain abdomen. On clinical examination, she was icteric, had tenderness in vertebral level L2, L3 and in left scapular region. On pet scan she was diagnosed to have multiple skeletal mets, left paraaortic, abdominopelvic lymph nodes and liver mets. The patient had a significantly deranged liver function test, and low haemoglobin levels so she was kept on supportive care and before any palliative treatment could be given, she died of the disease around 14 months after diagnosis.

\section{Discussion}

Primary small cell neuroendocrine carcinoma of female genital tract account for less than $2 \%$ of all gynaecological malignancies [1,2]. Scully, et al. reported the first case of primary small cell neuroendocrine carcinoma of vagina in $1984[1,4]$. Rarity of the tumour can be understood by the fact that till date only 26 cases have been registered in the literature $[1,4,5]$. Clinical profile of patients varies from being asymptomatic to having localized abdominal pain, vaginal bleeding, abdominal bloating or a vaginal mass. These tumors can be either functional or non-functional. Most common age of presentation is around 50-65 years and median survival is 11 months ranging from 4 months to 24 months. These tumors typically have aggressive behaviour and poor prognosis and present with distant metastasis in most cases. Irrespective of clinical stage of presentation, most patients die eventually due to distant metastasis. Owing to the rarity of the tumour, there is a lack of prospective randomized control trials resulting in no standard treatment protocols. A multimodality treatment strategy is recommended, keeping in view the aggressive nature of the disease. Surgery is advocated for cases with small, localized tumors without metastatic disease. Treatment of this tumour is done considering the general guidelines of management for small cell carcinomas of other sites. Patients are managed aggressively with combination chemotherapy of cisplatin and etoposide with radiation therapy including external beam radiotherapy to pelvis up to 45 Gy followed by brachytherapy [6]. Three chemotherapy regimens are usually used for combination chemotherapy, which includes vincristine, adriamycin and cyclophosphamide/cisplatin and etoposide or cyclophosphamide, adriamycin [7]. Topotecan, Paclitaxel and Bevacizumab combination regimen have also been shown to have a significant role in recurrent small cell neuroendocrine cancer of vagina [8]. Recently, gene expression testing is being advocated in order to design customized treatment regimens depending on the characteristics of the tumor. Gene expression testing is designed to specifically determine the expression levels of selected biomarkers within formalin-fixed paraffin-embedded tumor samples. Biomarkers selected for testing are based on extensive literature that strongly supports the gene as a biomarker for a specific drug and for the response 
to that drug in a specific tumor type [9]. There is lack of specific guidelines for the treatment of such rare malignancies. An aggressive multimodality treatment is recommended even for early-stage disease.

\section{References}

1. V. Singh, H. Singh, C. Leong, R. Hwang, and W Noquera (2008) Vaginal small cell carcinoma: Case report and review of literature. New York Med J.

2. WA Peters III, NB Kumar, GW Morley (1985) Carcinoma of the vagina. Factors influencing treatment outcome. Cancer 55: 892-897.

3. CJ Prasad, JA Ray, S Kessler (1992) Primary small cell carcinoma of the vagina arising in a background of atypical adenosis. Cancer 70: 2484-2487.

4. Z Bing, L Levine, JA Lucci, SS Hatch, MA Eltorky (2004) Primary small cell neuroendocrine carcinoma of the vagina: A clinicopathologic study. Arch Pathol Lab Med 128: 857862.
5. RE Joseph, MH Enghardt, DL Doering, BF Brown, DW Shaffer, et al. (1992) Small cell neuroendocrine carcinoma of the vagina. Cancer 70: 784-789.

6. Moertel C, Kvols L, O'Connell M, Rubin J (1991) Treatment of neuroendocrine carcinomas with combined etoposide and cisplatin: Evidence of major therapeutic activity in the anaplastic variants of these neoplasms. Cancer 68: 227232.

7. Hayashi M, Mori $\mathrm{Y}$, Takagi $\mathrm{Y}$, Hoshimoto $\mathrm{K}$, Ohkura $\mathrm{T}$ (2000) Primary small cell neuroendocrine carcinoma of the vagina. Oncology 58: 300-304.

8. M Frumovitz, MF Munsell, JK Burzawa, LA Byers, P Ramalingam, et al. (2017) Combination therapy with topotecan, paclitaxel, and bevacizumab improves progression-free survival in recurrent small cell neuroendocrine carcinoma of the cervix. Gynecol Oncol 144: 46-50.

9. Kostamo K, Peart M, McKenzie N, Holloman C, Carlan SJ, et al. (2018) Novel treatment of small-cell neuroendocrine of the vagina. Case Rep Oncol Med. 